\title{
All-Optical Reconfigurable Metamaterial Employing the Self-Assembly of CdTe Quantum Dots
}

\author{
Juan Adrian Zepeda-Galvez', Daniel Shreiber ${ }^{2}$, Arturo Ayon ${ }^{1}$ \\ ${ }^{1}$ The University of Texas at San Antonio, San Antonio, TX, USA \\ ${ }^{2}$ CCDC Army Research Laboratory, FCDD-RLW-ME, Aberdeen Proving Ground, MD, USA \\ Email: juanadrian.zepeda-galvez@utsa.edu
}

How to cite this paper: Zepeda-Galvez, J.A., Shreiber, D. and Ayon, A. (2020) All-Optical Reconfigurable Metamaterial Employing the Self-Assembly of CdTe Quantum Dots. Open Journal of Inorganic Non-metallic Materials, 10, 31-43.

https://doi.org/10.4236/ojinm.2020.103003

Received: July 3, 2020

Accepted: July 28, 2020

Published: July 31, 2020

Copyright $\odot 2020$ by author(s) and Scientific Research Publishing Inc. This work is licensed under the Creative Commons Attribution International License (CC BY 4.0).

http://creativecommons.org/licenses/by/4.0/

\section{(c) (i) Open Access}

\begin{abstract}
Positively and negatively charged polyelectrolytes, namely, Poly(diallyldimethylammonium chloride) and Poly(styrene sulfonate), respectively, were employed to disperse and deploy negatively charged quantum dots on an otherwise passive metamaterial structure with a resonant frequency of $0.62 \mathrm{THz}$, by employing a layer-by-layer, self-assembly scheme. Upon exposure to a UV source with a wavelength of $365 \mathrm{~nm}$ the amplitude modulation was observed to increase with increases in the number of deposited bi-layers, until a modulation maximum of $2.68 \%$ was recorded enabling an all-optical, dynamically reconfigurable metamaterial geometry. Furthermore, amplitude modulation was subsequently observed to decrease with further increases in the number of layers employed due to quenching and shadowing effects. The experimental observations reported herein will enable the utilization of all-optical reconfigurable $\mathrm{THz}$ devices for communication and data transmission applications.
\end{abstract}

\section{Keywords}

Metamaterials, Quantum Dots, CdTe, Terahertz, Reconfigurable Devices

\section{Introduction}

The ability to control the amplitude, phase and/or polarization of electromagnetic waves remains of critical importance for all technological applications. This is also applicable to Terahertz (THz) radiation, ranging from $100 \mathrm{GHz}$ to $5 \mathrm{THz}$ that is of interest to scientists and technologists because electromagnetic waves at those frequencies can excite intra- and intermolecular resonances due to mo- 
lecular rotations or vibrations and Van de Waals forces [1] [2]. Additionally, $\mathrm{THz}$ electromagnetic waves exhibit a variety of useful characteristics, namely, $\mathrm{THz}$ photons are non-ionizing and most dielectrics are transparent to them, enabling a wide range of applications, from characterization of chemical substances and quality control [3] [4] [5] [6] [7] to contributions in communications, and conservation of cultural heritage [8] [9] [10] [11]. In terms of the size of the objects under consideration, it extends from the galactic scale to nanoscale systems [12] [13] [14] [15] [16]. Interestingly, THz devices are, in general, faster and more sensitive, compared to their counterparts in other regions of the electromagnetic spectrum [17]; however, the paucity of naturally occurring materials with a suitable performance in the $\mathrm{THz}$ regime has made the advance of terahertz devices and applications rather arduous. This shortcoming can be addressed by the judicious utilization of metamaterials, which are artificial media with subwavelength unit cells that can be designed to achieve arbitrary (negative or positive) permittivity and permeability values, and can be custom-tailored to exhibit resonances at a desired frequency even in the THz regime [18] [19] [20] [21] [22]. The resonance amplitude and frequency of operation of metamaterials are determined by the geometry and the materials utilized, and typically do not exhibit a reconfigurable behavior, which is a requirement in numerous applications. A variety of schemes have been suggested and explored to achieve reconfigurable metamaterial performance including the dynamic adjustment of the dimensions or the spacing of the structures which can be effected either by employing flexible surfaces [23] [24] or microactuating mechanisms frequently associated with MEMS technology [25] [26]. The use of phase-change materials, either thermal, electrical or electromagnetically controlled, can also attain such a goal [27] [28]. However, an all-optical reconfigurable device is highly desirable for $\mathrm{THz}$ communication and data transmission applications, and a dynamic reconfigurable performance could be achieved by modulating the dielectric characteristics of the media surrounding the metamaterial unit cell, for instance by the utilization of semiconducting quantum dots (QDs). Specifically, it has recently been reported that Cadmium Telluride (CdTe) QDs exhibit a variation in their dielectric characteristics when they are excited by an ultraviolet (UV) source [29]. However, their ability to modulate the response of a metamaterial array operating in the $\mathrm{THz}$ regime has not been demonstrated yet.

\section{Experimental Details}

\subsection{Quantum Dot Synthesis}

The CdTe QDs employed as reconfigurable elements, were obtained by a hydrothermal synthesis method. In a typical synthesis, $0.0533 \mathrm{~g}(0.2 \mathrm{mmol})$ of Cadmium acetate dihydrate $\left(\mathrm{Cd}\left(\mathrm{CH}_{3} \mathrm{COO}\right)_{2} 2 \mathrm{H}_{2} \mathrm{O}, 99.5 \%\right)$ were dissolved in 50 $\mathrm{ml}$ of deionized (DI) water in a $125-\mathrm{ml}$ Erlenmeyer flask. Next, $18 \mu \mathrm{l}$ of thioglycolic acid (TGA) were added and $1 \mathrm{M} \mathrm{NaOH}$ solution was incorporated dropwise to adjust the $\mathrm{pH}$ to a value between 10.5 - 11. In a separate flask, $0.0101 \mathrm{~g}$ 
$(0.04 \mathrm{mmol})$ of potassium tellurite $\left(\mathrm{K}_{2} \mathrm{TeO}_{3}, 95 \%\right)$ were dissolved in $50 \mathrm{ml}$ of DI water and added to the first solution. Subsequently, $0.08 \mathrm{~g}$ of sodium borohydride $\left(\mathrm{NaBH}_{4}, 99.99 \%\right)$ was added to the mixture and the reaction was allowed to stir for $5 \mathrm{~min}$. The solution was transferred to a three-neck round flask and attached to a Liebig condenser and refluxed under atmospheric conditions, at a temperature of $100^{\circ} \mathrm{C}$. QD size is determined by the reflux time. The solution was then washed with acetone and redispersed in deionized water to obtain a clean colloidal solution. A high-resolution TEM micrograph of the synthesized QDs is shown in the inset of Figure 1(b). Since the QDs are capped with TGA, their surfaces contain negatively-charged carboxylate groups [30] [31]. This makes CdTe QDs a suitable candidate for a layer-by-layer deposition employing positively and negatively charged polyelectrolytes, namely, Poly(diallyldimethylammonium chloride) and Poly(styrene sulfonate), respectively.

\subsection{Quantum Dot Characterization}

Quantum dot optical absorption characterization was performed employing an Ocean Optics Flame-S-UV-VIS spectrometer (Figure 1(a)). Bandgap energy and absorption are related by the following expression [32]

$$
\alpha h v=A\left(h v-E_{g}\right)^{(1 / r)}
$$

where $\alpha$ is the experimentally collected absorption, $h$ is Planck's constant, $v$ is the photon's frequency, $E_{g}$ is the quantum dot bandgap energy and $A$ is a proportionality constant. The nominal value of $r$ reflects the nature of the optical transition. For a direct allowed transition, $r$ is set to be 2 . The optical bandgap can then be estimated graphically employing a method described by Tauc [33] (Figure 1(b)). The extracted bandgap value of CdTe QDs was estimated to be $3.11 \mathrm{eV}$. Alternatively, after a mathematical manipulation, the above equation can be rewritten as [34]

$$
\frac{\mathrm{d}(\ln (\alpha h v))}{\mathrm{d}(h v)}=\frac{1}{r\left(h v-E_{g}\right)}
$$

According to the above equation, a plot of $\mathrm{d}(\ln (\alpha h v)) / \mathrm{d}(h v)$ vs. $h v$ should exhibit a discontinuity when $h v-E_{g} \rightarrow 0$. However, due to impurities and QD size inhomogeneity, the discontinuity could be replaced by a maximum. Figure 2 illustrates this method, from which a bandgap value of $3.18 \mathrm{eV}$ was obtained, in reasonable agreement with the previously estimated value employing Tauc's graphic method.

Additionally, QD size can be estimated using Brus' quantum mechanical approximation [35]

$$
E_{Q D}=E_{B u l k}+\frac{\hbar^{2} \pi^{2}}{2 r^{2}}\left(\frac{1}{m_{e}^{*}}+\frac{1}{m_{h}^{*}}\right)-\frac{1.8 \mathrm{e}^{2}}{4 \pi \epsilon \epsilon_{0} r}
$$

where $r$ is the radius, $\epsilon=10.2$ is the relative permittivity of bulk CdTe, $m_{e}^{*}=0.11 m_{0}$ and $m_{h}^{*}=0.4 m_{0}$ are the respective electron and hole effective 

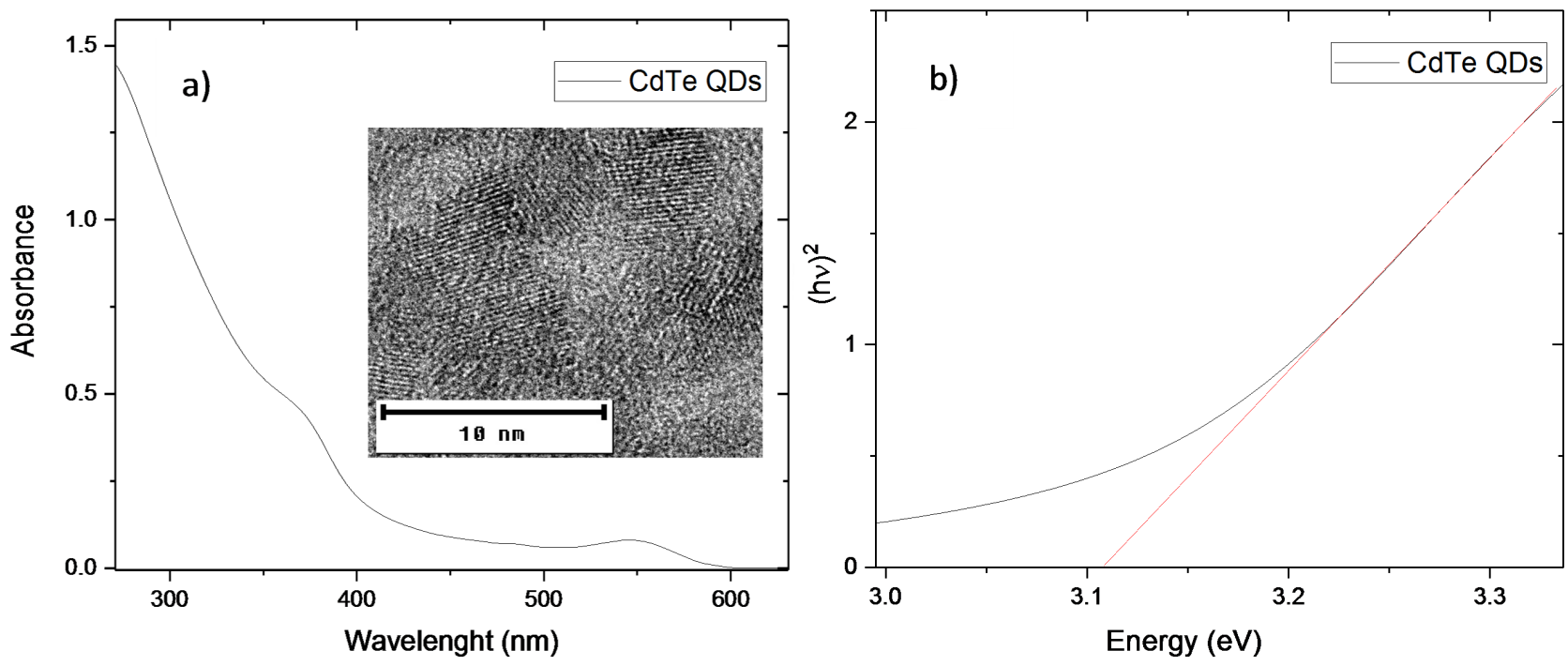

Figure 1. (a) CdTe QDs optical absorption; (b) Tauc plot showing the estimated optical bandgap at $3.11 \mathrm{eV}$. The inset in (a) is an HR TEM of the QDs.

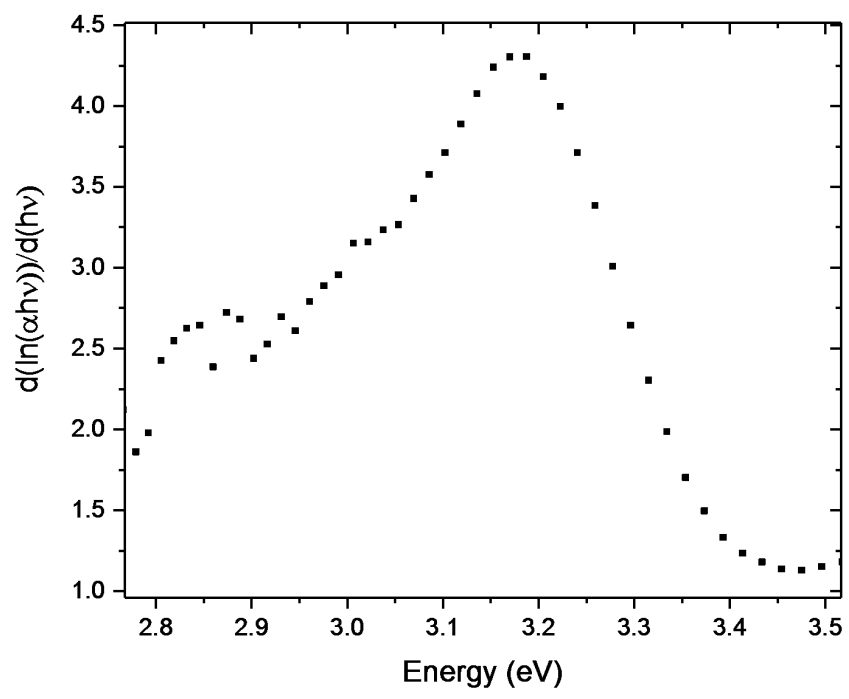

Figure 2. Plot of $\mathrm{d}(\ln (\alpha h v)) / \mathrm{d}(h v)$ vs. $h v$ showing the bandgap value at the maximum position.

masses, $e$ is the electron charge, and $E_{Q D}$ and $E_{B u l k}$ are the QD and bulk bandgap values, respectively. Utilizing these parameters, the radius of the synthesized QDs was estimated to be $1.56 \mathrm{~nm}$. Moreover, CdTe QDs size can also be approximated by the empirical equation [30]

$$
D=\left(9.8127 \times 10^{-7}\right) \lambda^{3}-\left(1.7147 \times 10^{-3}\right) \lambda^{2}+(1.0064) \lambda-194.84
$$

where $D$ is the diameter of the QDs and $\lambda$ is the wavelength of the first excitonic absorption peak. As shown in Figure 1(a), the first excitonic absorption peak is located at a wavelength of $540 \mathrm{~nm}$, indicating a diameter of $3.2 \mathrm{~nm}$, in close agreement to the quantum mechanical approach. The confinement state of the generated electron-hole pairs can be determined by comparing the previous val- 
ue to the Bohr radius corresponding to the first excitonic state which is given by the equation [36]

$$
r_{B}=\frac{\epsilon \epsilon_{0} h^{2}}{\pi \mathrm{e}^{2}}\left(\frac{1}{m_{e}^{*}}+\frac{1}{m_{h}^{*}}\right)
$$

Since the calculated Bohr radius for the first excitonic state is $7.5 \mathrm{~nm}$ for $\mathrm{CdTe}$, and, therefore, larger than the size of the synthesized QDs, it evinces the strong confinement state of electrons and holes [37].

In order to conduct $\mathrm{THz}$ measurements employing colloidal quantum dots, water must be exchanged as the host solvent, due to its extremely high absorption coefficient [38]. A thiol exchange scheme was employed to make the QDs solvable on toluene, which has a much lower THZ absorption coefficient [39], [40]. To this end, $1 \mathrm{ml}$ of 1-dodecanethiol was added to $1 \mathrm{ml}$ of a clean solution of CdTe QDs. Subsequently, $2 \mathrm{ml}$ of acetone were added to the mixture followed by 2 minutes of vortexing. The solution was then heated to $60^{\circ} \mathrm{C}$ until the organic phase was reduced to $1 \mathrm{ml}$. After the QDs had been transferred to the organic phase, they were diluted with toluene and precipitated with methanol. The product was decanted and diluted once more with toluene.

Quantum dot concentration was measured employing a quartz crystal microbalance (QCM) instrument. The technique is based on the resonance frequency shift of a piezoelectric crystal. The deposition of a liquid drop of colloidal QDs on top of an oscillating quartz crystal provokes a shift in its resonance frequency, which is related to the mass of the residual QDs film after the solvent was evaporated by the following equation [41]

$$
\Delta m=\frac{\Delta f}{C_{f}}
$$

where $\Delta f$ is the frequency shift and $C_{f}$ is the crystal sensitivity factor (56.6 $\left.\mathrm{Hz} \cdot \mathrm{\mu g}^{-1} \cdot \mathrm{cm}^{2}\right)$. The concentration of CdTe QDs dissolved in toluene was measured by dropping $15 \mu \mathrm{l}$ of solution on the crystal's active area. After the solvent was evaporated, the residual mass was measured. This process was repeated 9 times and the results were linearly fitted to obtain the concentration in units of $\mu \mathrm{g} / \mu \mathrm{l}$ (see Figure 3).

Quantum Dot Terahertz characterization was carried out by employing a $\mathrm{THz}$ time domain spectroscopy (THz-TDS) technique, operating in transmission mode. The system makes use of a photoconductive antenna to generate an ultrafast, broadband $\mathrm{THz}$ pulse when is activated by a femtosecond laser with a wavelength of $800 \mathrm{~nm}$. A second photoconductive antenna is utilized as a detector. Once proper alignment was achieved, the system was thermalized for 2 hours. An ultraviolet lamp $\left(365 \mathrm{~nm}, 20 \mathrm{~mW} / \mathrm{cm}^{2}\right)$ was employed to excite the sample.

The time domain Terahertz signal is transformed into the frequency domain by a Fast Fourier Transform to obtain the amplitude and phase change. Air and the empty cuvette were used as references to calculate the complex permittivity 


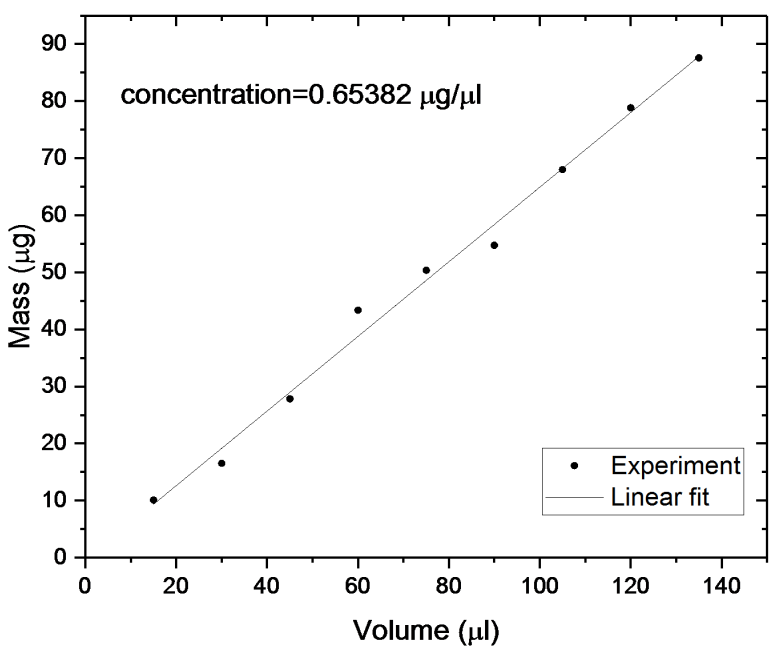

Figure 3. Mass of the QDs film as a function of the volume dropped on top of the QCM crystal. The slope of the linear fit represents QD concentration

of toluene and colloidal quantum dots [42]. Subsequently, linear effective medium theory was employed to obtain the permittivity of the Quantum dots through the relation [43]

$$
\epsilon_{\text {mix }}=c \epsilon_{Q D s}+(1-c) \epsilon_{h}
$$

where $c$ is the volume fraction of colloidal QDs and $\epsilon_{h}$ is the permittivity of the host solvent, which, for CdTe QDs was toluene. Figure 4 shows the real and imaginary part of the permittivity of the QDs, after applying the aforementioned effective medium theory. Ostensibly, the real and imaginary parts of $\epsilon$ increase when the nanoparticles are excited by an ultraviolet source. This change is thought to be related to the large exciton polarizability of the QDs, due to their atom-like response, which is present in the strong confinement state [44].

\subsection{Metamaterial Simulation}

Finite Difference Time-Domain (FDTD) simulations were performed in order to elucidate the electromagnetic response of the metamaterial geometry. This technique solves Maxwell's equations numerically in discretized time and space and the results are the electric and magnetic field distributions following the interaction of the source fields with the material distribution. The simulations were carried out employing a commercial solver (Lumerical Inc., ND). The dimensions of the simulated metamaterial unit cell are shown in Figure 5(a) employing a design reported elsewhere [45]. The outer black hexagon represents the boundary of the unit cell and the red lines represent a Perfect Electric Conductor (PEC).

The metallic incursions are placed on top of a silicon slab with a refractive index of $n=3.42+0.01 i$ [46]. Periodical boundary conditions were set in the directions orthogonal to the electromagnetic source, and a Perfectly Matched Layer (PML) was set in the direction parallel to it. The electromagnetic source 


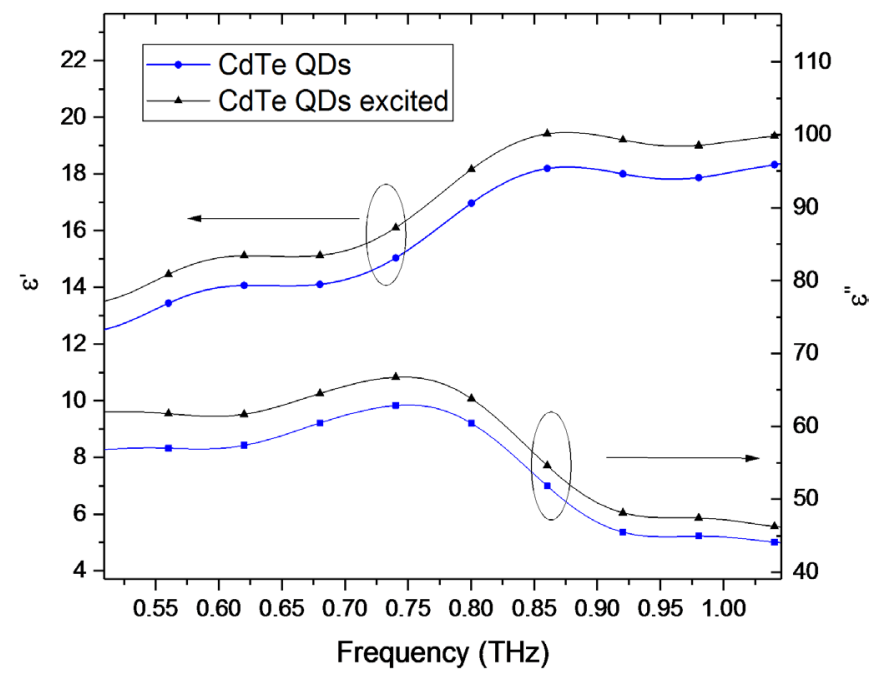

Figure 4. Spectral dependence of the real and imaginary parts of the complex permittivity. The traces in blue correspond to the colloidal samples without UV excitation, while the traces in black correspond to the same samples under UV excitation with a wavelength of $365 \mathrm{~nm}$.
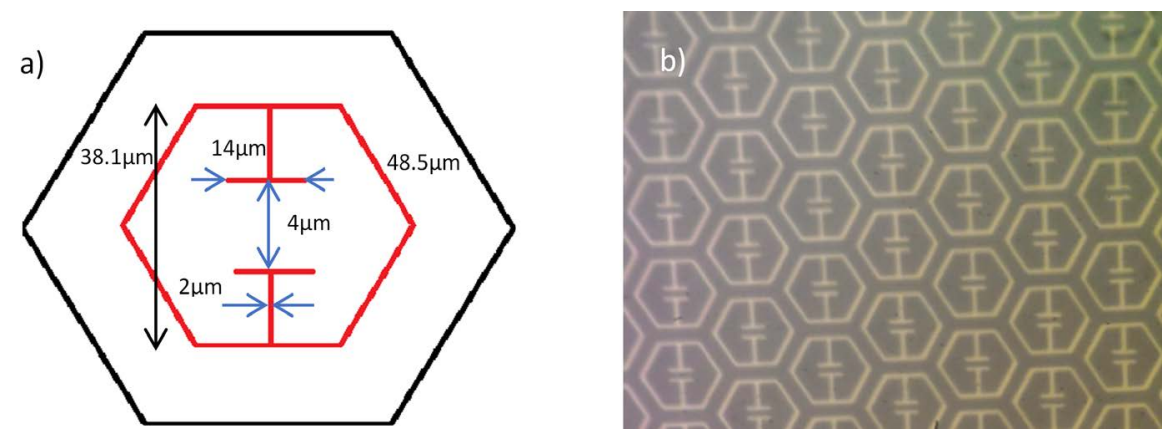

Figure 5. (a) Diagram of the simulated unit cell; (b) Optical microscope image of the fabricated metamaterial.

was a plane wave with the electric field aligned orthogonal to the capacitor plates in the center of the unit cell. An optical image of the microfabricated metamaterial structure is shown in Figure 5(b).

Figure 6 shows the simulated and experimental transmission graphs featuring a resonance at $0.62 \mathrm{THz}$. The electric field distribution at the resonant frequency is illustrated in Figure 6(b). Ostensibly, the electric field is strongly confined within the capacitor area of the unit cell.

\subsection{Metamaterial Fabrication}

For Metamaterial fabrication a standard image reversal lift-off procedure was performed on a $<100>$ high resistivity silicon substrate, with a nominal thickness of $400 \mu \mathrm{m}$. Silver was chosen as the material for the metallic array due to its relatively low-loss properties [47]. To this end, thermal evaporation was employed to deposit a silver layer with a thickness of $100 \mathrm{~nm}$ on top of a $20 \mathrm{~nm}$ layer of 

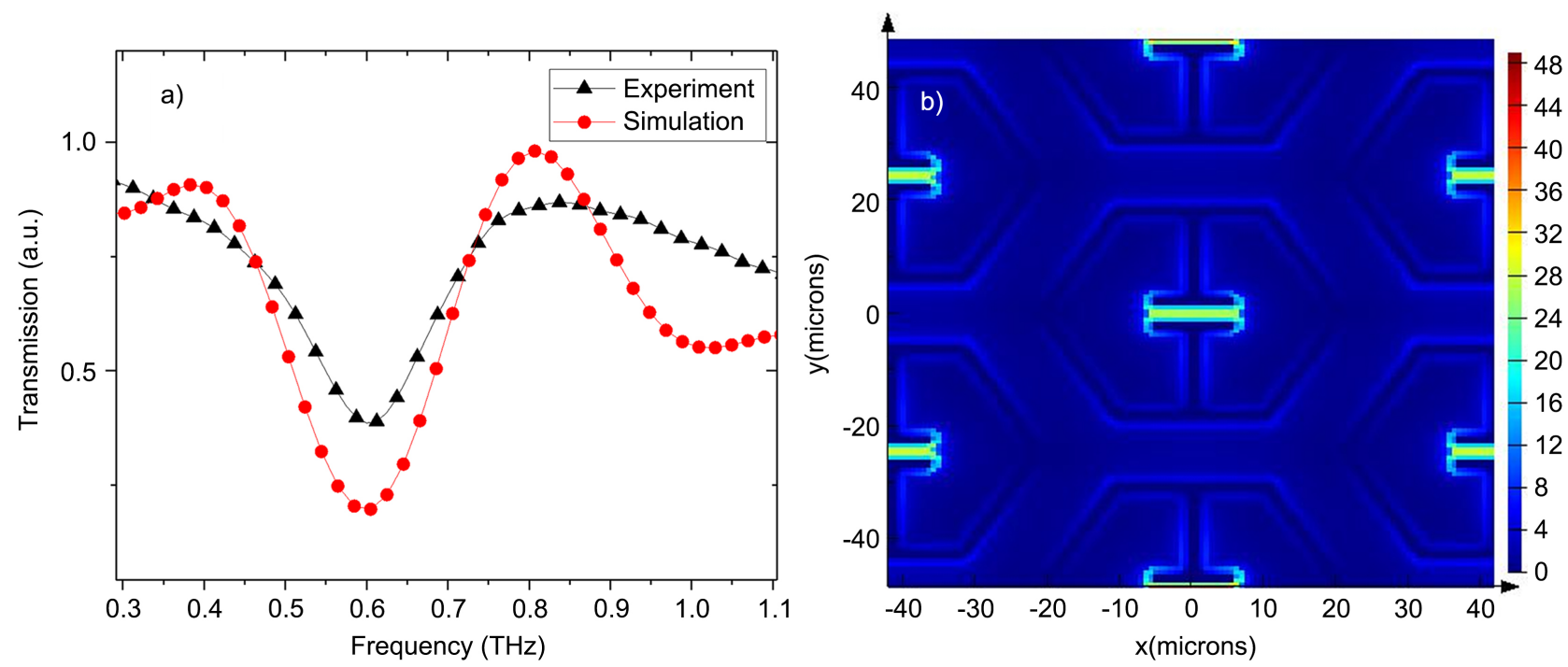

Figure 6. (a) Resonant frequency value from computational simulation and experimental observation; (b) Electric field distribution at $0.6 \mathrm{THz}$, featuring an electric field confinement at the capacitor area.

chromium that was included for adhesion purposes. Terahertz Time-Domain Spectroscopy (THz TDS) in transmission mode was employed to experimentally explore the resonant properties of the metamaterial. The measurements were performed at room temperature with a Menlo Systems TeraSmart K15 equipment. Five thousand waveforms were averaged for each sample, and the time domain waveforms were passed through a Gaussian window to remove the Fabry-Perot oscillations in order to obtain a cleaner signal [48]. Subsequently, a Fast Fourier Transform (FFT) was applied to obtain the amplitude and phase of the transmitted beam as a function of frequency. In order to extract the effect of the metamaterial, we define the transmission as the ratio of the amplitude of the electric field through the metamaterial structure to the one through a blank, 400 um thick silicon wafer. Prior to deploying the synthesized QDs, the substrate with the metamaterial geometry was exposed to an $\mathrm{O}_{2}$ plasma $(30 \mathrm{~W}$ for $10 \mathrm{mi}-$ nutes) to activate the surface, and two bilayers of alternating positively charged Poly(diallyldimethylammonium chloride) (PDDA) (1.5 wt\%) and negatively charged Poly(styrene sulfonate) (PSS) (0.3 wt\%) were spin coated at $2500 \mathrm{rpm}$ for $2 \mathrm{~min}$. Subsequently, CdTe QDs were then deployed on the metamaterial by employing a bilayer scheme (Figure 7) [49] in which a layer of PDDA was spin cast on the surface, followed by a layer of the aqueous solution of negatively charged CdTe QDs. This last alternating bilayer step was repeated 6, 10, 13 and 15 times to evaluate the performance as a function of the number of bilayers employed. The sample surface was rinsed with DI water and dried with a gentle stream of $\mathrm{N}_{2}$ after each individual spin coated layer. Profilometry measurements indicated that, on average, for every pair of PDDA + CdTe QDs layers, the thickness increased by approximately $10 \mathrm{~nm}$.

Transmission modulation (TM) was measured employing an ultraviolet source with a wavelength of $365 \mathrm{~nm}$, which corresponds to an energy of $3.4 \mathrm{eV}$, 
i.e., larger than the bandgap energy of CdTe QDs. The UV source was set to illuminate all samples with a different number of bilayers while varying the incident power density from 6 to $20 \mathrm{~mW} / \mathrm{cm}^{2}$. At the resonant frequency of 0.62 $\mathrm{THz}$, the TM was observed to increase with all incident power density increases (Figure $8(\mathrm{a})$ ). This is thought to be due to the susceptibility of the photoexcited QDs dependence on the excitation density. That is, the number of incident photons per unit area has an impact on the polarizability of the excitons confined in the QDs [44].

In terms of the number of bilayers employed, the TM value increased as the number of bilayers increased from 6 to 10 and decreased for subsequent increases in the number of bilayers, i.e., 13 and 15 bilayers. Specifically, for 6 bilayers, the modulation increased from $0.2 \%$ to $1.3 \%$, the modulation observed

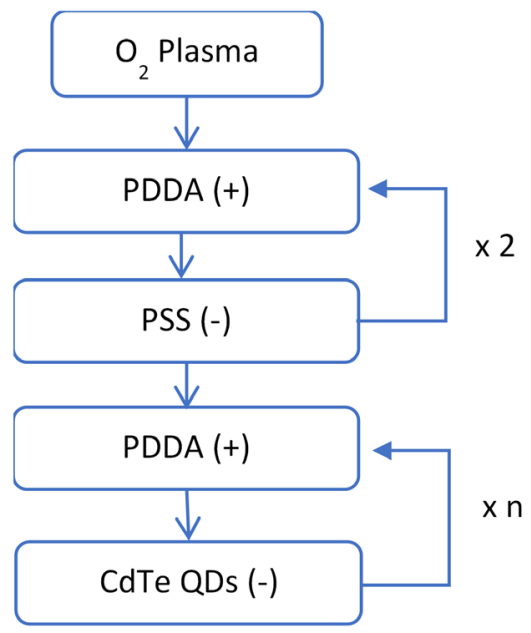

Figure 7. Process flow illustrating the layer-by-layer method described herein.
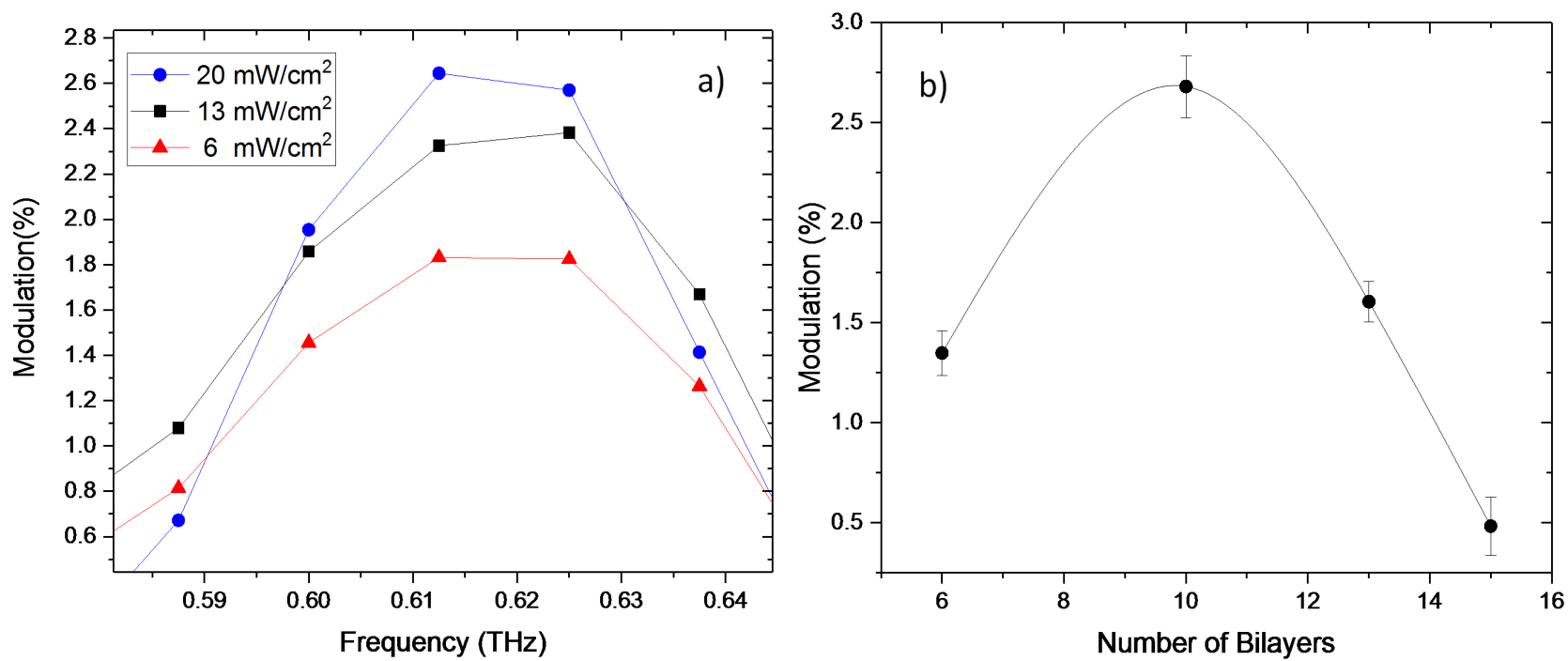

Figure 8. (a) Modulation as a function of frequency for the metamaterial with 10 bilayers; (b) Modulation at $0.62 \mathrm{THz}$ for the highest power density as a function of number of layers. 
with 10 bilayers increased from $1.84 \%$ to $2.68 \%$, and with 13 bilayers, the modulation increased from $1.2 \%$ to $1.6 \%$. Lastly, for 15 bilayers, the modulation increased from $0.13 \%$ to $0.48 \%$, being the smallest modulation attained. Quenching and shadowing effects are thought to be responsible for the observed decrease in transmission modulation depth when employing 13 or more bilayers which is presented in Figure 8(b).

\section{Conclusion}

Colloidal CdTe QDs were chemically synthesized and utilized as a reconfigurable element in a previously fabricated metamaterial structure that exhibited a resonance frequency at $0.62 \mathrm{THz}$. Modulation due to an ultraviolet source with different power densities and different numbers of polyelectrolyte bilayers were probed. The experimental data revealed that the modulation is largest when 10 layers of QDs + PDDA are deployed and the maximum modulation achieved was $2.68 \%$, with a power density reaching $20 \mathrm{~mW} / \mathrm{cm}^{2}$. Furthermore, the proximity to a linear relation suggests that a larger modulation can be achieved at higher power densities. Additionally, the entire process is mass production compatible and can be integrated with other electronic circuitry microfabrication processes, making it attractive for a variety of technological applications

\section{Acknowledgements}

This work was supported by the Air Force Office of Scientific Research (AFOSR) Grant number FA9550-17RYCOR490.

\section{Conflicts of Interest}

The authors declare no conflicts of interest regarding the publication of this paper.

\section{References}

[1] Semenova, V. and Bespalov, V. (2015) Terahertz Technologies for Telecommunications. Photonics, 51, 126-141.

[2] Dhillon, S.S., et al. (2017) The 2017 Terahertz Science and Technology Roadmap. Journal of Physics D: Applied Physics, 50, Article ID: 043001.

[3] Ajito, K. and Ueno, Y. (2011) THz Chemical Imaging for Biological Applications. IEEE Transactions on Terahertz Science and Technology, 1, 293-300. https://doi.org/10.1109/TTHZ.2011.2159562

[4] Karpowicz, N., et al. (2005) Compact Continuous-Wave Subterahertz System for Inspection Applications. Applied Physics Letters, 86, Article ID: 054105. https://doi.org/10.1063/1.1856701

[5] Kumar, C.S.S.R. (2013) UV-VIS and Photoluminescence Spectroscopy for Nanomaterials Characterization.

[6] Wietzke, S., Jördens, C., Krumbholz, N., Baudrit, B., Bastian, M. and Koch, M. (2007) Terahertz Imaging: A New Non-Destructive Technique for the Quality Control of Plastic Weld Joints. Journal of the European Optical Society, 2, 2-6. 
https://doi.org/10.2971/jeos.2007.07013

[7] Pickwell, E. and Wallace, V.P. (2006) Biomedical Applications of Terahertz Technology. Journal of Physics D: Applied Physics, 39, R301.

https://doi.org/10.1088/0022-3727/39/17/R01

[8] Gomez-Sepulveda, A.M., et al. (2017) History of Mexican Easel Paintings from an Altarpiece Revealed by Non-Invasive Terahertz Time-Domain Imaging. Journal of Infrared, Millimeter, and Terahertz Waves, 38, 403-412. https://doi.org/10.1007/s10762-016-0346-x

[9] Nagatsuma, T., Ducournau, G. and Renaud, C.C. (2016) Advances in Terahertz Communications Accelerated by Photonics. Nature Photonics, 10, 371-379. https://doi.org/10.1038/nphoton.2016.65

[10] Lin, C. and Li, G.Y.L. (2016) Terahertz Communications: An Array-of-Subarrays Solution. IEEE Communications Magazine, 54, 124-131. https://doi.org/10.1109/MCOM.2016.1600306CM

[11] Oshima, N., Hashimoto, K., Suzuki, S. and Asada, M. (2017) Terahertz Wireless Data Transmission with Frequency and Polarization Division Multiplexing Using Resonant-Tunneling-Diode Oscillators. IEEE Transactions on Terahertz Science and Technology, 7, 593-598. https://doi.org/10.1109/TTHZ.2017.2720470

[12] Leisawitz, D.T., et al. (2000) Scientific Motivation and Technology Requirements for the SPIRIT and SPECS Far-Infrared/Submillimeter Space Interferometers. Proceedings UV, Optical, and IR Space Telescopes and Instruments, Volume 4013, 36-46. https://doi.org/10.1117/12.393957

[13] Chamorro-Posada, P., et al. (2016) THz TDS Study of Several sp2 Carbon Materials: Graphite, Needle Coke and Graphene Oxides. Carbon N. Y., 98, 484-490. https://doi.org/10.1016/j.carbon.2015.11.020

[14] Leyman, R.R., et al. (2016) Quantum Dot Materials for Terahertz Generation Applications. Laser \& Photonics Reviews, 10, 772-779.

https://doi.org/10.1002/lpor.201500176

[15] Mandal, P.K. and Chikan, V. (2007) Plasmon-Phonon Coupling in Charged n-Type CdSe Quantum Dots: A THz Time-Domain Spectroscopic Study. Nano Letters, 7, 2521-2528. https://doi.org/10.1021/n1070853q

[16] Phillips, T.G. and Keene, J. (1992) Submillimeter Astronomy (Heterodyne Spectroscopy). Proceedings of the IEEE, 80, 1662-1678. https://doi.org/10.1109/5.175248

[17] Nasa, S. and Purohit, S.P. (2020) Linear and Third Order Nonlinear Optical Properties of GaAs Quantum Dot in Terahertz Region. Physica E: Low-Dimensional Systems and Nanostructures, 118, Article ID: 113913. https://doi.org/10.1016/j.physe.2019.113913

[18] Costa, F., Monorchio, A. and Manara, G. (2010) Analysis and Design of Ultra Thin Electromagnetic Absorbers Comprising Resistively Loaded High Impedance Surfaces. IEEE Transactions on Antennas and Propagation, 58, 1551-1558. https://doi.org/10.1109/TAP.2010.2044329

[19] Zarei, S., Mataji-Kojouri, A., Shahabadi, M. and Mohajerzadeh, S. (2018) Tunable Terahertz Filter Composed of an Array of Subwavelength Metallic Ring Apertures. Optik (Stuttg), 164, 355-361. https://doi.org/10.1016/j.ijleo.2018.03.015

[20] Baena, J.D., et al. (2005) Equivalent-Circuit Models for Split-Ring Resonators and Complementary Split-Ring Resonators Coupled to Planar Transmission Lines. IEEE Transactions on Microwave Theory and Techniques, 53, 1451-1460. https://doi.org/10.1109/TMTT.2005.845211 
[21] Ye, W., et al. (2016) Spin and Wavelength Multiplexed Nonlinear Metasurface Holography. Nature Communications, 7, Article No. 11930. https://doi.org/10.1038/ncomms11930

[22] Vázquez-Colón, C.D., Zepeda-Galvéz, J.A. and Ayón, A.A. (2019) Reconfigurable X-Shaped THz Metamaterials Employing Quantum Dots. Symposium on Design, Test, Integration \& Packaging of MEMS and MOEMS, Paris, 12-15 May 2019, 1-2. https://doi.org/10.1109/DTIP.2019.8752863

[23] Lee, S., et al. (2012) Reversibly Stretchable and Tunable Terahertz Metamaterials with Wrinkled Layouts. Advanced Materials, 24, 3491-3497. https://doi.org/10.1002/adma.201200419

[24] Li, J., et al. (2013) Mechanically Tunable Terahertz Metamaterials. Applied Physics Letters, 102, 2011-2015. https://doi.org/10.1063/1.4773238

[25] Han, Z., Kohno, K., Fujita, H., Hirakawa, K. and Toshiyoshi, H. (2015) Tunable Terahertz Filter and Modulator Based on Electrostatic MEMS Reconfigurable SRR Array. IEEE Journal of Selected Topics in Quantum Electronics, 21, 114-122. https://doi.org/10.1109/JSTQE.2014.2378591

[26] Zhu, W.M., et al. (2011) Polarization Dependent State to Polarization Independent State Change in THz Metamaterials. Applied Physics Letters, 99, 10-13. https://doi.org/10.1063/1.3664131

[27] Zhao, Y., et al. (2018) Dynamic Photoinduced Controlling of the Large Phase Shift of Terahertz Waves via Vanadium Dioxide Coupling Nanostructures. ACS Photonics, 5, 3040-3050. https://doi.org/10.1021/acsphotonics.8b00276

[28] Wu, L., et al. (2016) A New $\mathrm{Ba}_{0.6} \mathrm{Sr}_{0.4} \mathrm{TiO}_{3}$-Silicon Hybrid Metamaterial Device in Terahertz Regime. Small, 12, 2610-2615. https://doi.org/10.1002/smll.201600276

[29] Vázquez-colón, C.D., Zepeda, J.A., Ayón, A. and Shreiber, D. (2017) Reconfigurable Behavior of CdTe Quantum Dots at Terahertz Frequencies Employing UV Excitation. Symposium on Design, Test, Integration and Packaging of MEMS/MOEMS, Bordeaux, 29 May-1 June 2017, 4-6.

[30] Yu, W.W., Qu, L., Guo, W. and Peng, X. (2003) Experimental Determination of the Extinction Coefficient of CdTe, CdSe, and CdS Nanocrystals. Chemistry of Materials, 15, 2854-2860. https://doi.org/10.1021/cm034081k

[31] Wu, S., Dou, J., Zhang, J. and Zhang, S. (2012) A Simple and Economical One-Pot Method to Synthesize High-Quality Water Soluble CdTe QDs. Journal of Materials Chemistry, 22, 14573-14578. https://doi.org/10.1039/c2jm31409f

[32] Ibrahim, A. and Al-Ani, S.K.J. (1994) Models of Optical Absorption in Amorphous Semiconductors at the Absorption Edge-A Review and Re-Evaluation. Czechoslovak Journal of Physics, 44, 785-797. https://doi.org/10.1007/BF01700645

[33] Grigorovici, R., Vancu, A., et al. (1966) Optical Properties and Electronic Structure of Amorphous Germanium. Physica Status Solidi, 15, 627-637. https://doi.org/10.1002/pssb.19660150224

[34] Kar, S. and Chaudhuri, S. (2005) Synthesis and Optical Properties of Single and Bicrystalline ZnS Nanoribbons. Chemical Physics Letters, 414, 40-46. https://doi.org/10.1016/j.cplett.2005.08.021

[35] Brus, L.E. (1984) Electron-Electron and Electron-Hole Interactions in Small Semiconductor Crystallites: The Size Dependence of the Lowest Excited Electronic State. The Journal of Chemical Physics, 80, 4403-4409. https://doi.org/10.1063/1.447218

[36] Pokutnyi, S.I. (2012) Exciton States in Quasi-Zero-Dimensional Semiconductor Nanosystems. Semiconductors, 46, 165-170. 
https://doi.org/10.1134/S1063782612020194

[37] Flores-Pacheco, A., Álvarez-Ramos, M.E. and Ayón, A. (2020) Down-Shifting by Quantum Dots for Silicon Solar Cell Applications. In: Solar Cells and Light Management, Elsevier, Amsterdam, 443-477. https://doi.org/10.1016/B978-0-08-102762-2.00013-6

[38] Libon, I.H., et al. (1999) THz Spectroscopy of Polar Liquids. Proceedings Terahertz Spectroscopy and Applications, Vol. 3617, 24-29. https://doi.org/10.1117/12.347127

[39] D’Arco, A., et al. (2020) Characterization of Volatile Organic Compounds (VOCs) in Their Liquid-Phase by Terahertz Time-Domain Spectroscopy. Biomedical Optics Express, 11, 1. https://doi.org/10.1364/BOE.11.000001

[40] Gaponik, N., Talapin, D.V., Rogach, A.L. and Weller, H. (2002) Efficient Phase Transfer of Luminescent Thiol-Capped Nanocrystals: From Water to Nonpolar Organic Solvents. Nano Letters, 2, 803-806. https://doi.org/10.1021/nl025662w

[41] Reipa, V., Purdum, G. and Choi, J. (2010) Measurement of Nanoparticle Concentration Using Quartz Crystal Microgravimetry. The Journal of Physical Chemistry $B, 114,16112-16117$. https://doi.org/10.1021/jp103861m

[42] Yang, F., et al. (2017) Uncertainty in Terahertz Time-Domain Spectroscopy Measurement of Liquids. Journal of Infrared, Millimeter, and Terahertz Waves, 38, 229-247. https://doi.org/10.1007/s10762-016-0318-1

[43] Weissker, H.C., Furthmüller, J. and Bechstedt, F. (2003) Validity of Effective-Medium Theory for Optical Properties of Embedded Nanocrystallites from $A b$ Initio Supercell Calculations. Physical Review B: Condensed Matter and Materials Physics, 67, 1-5. https://doi.org/10.1103/PhysRevB.67.165322

[44] Wang, F., Shan, J., Islam, M.A., Herman, I.P., Bonn, M. and Heinz, T.F. (2006) Exciton Polarizability in Semiconductor Nanocrystals. Nature Materials, 5, 861-864. https://doi.org/10.1038/nmat1739

[45] Bingham, C.M., Tao, H., Liu, X., Averitt, R.D., Zhang, X. and Padilla, W.J. (2008) Planar Wallpaper Group Metamaterials for Novel Terahertz Applications. Optics Express, 16, 18565-18575. https://doi.org/10.1364/OE.16.018565

[46] Dai, J., Zhang, J., Zhang, W. and Grischkowsky, D. (2004) Terahertz Time-Domain Spectroscopy Characterization of the Far-Infrared Absorption and Index of Refraction of High-Resistivity, Float-Zone Silicon. Journal of the Optical Society of America $B, 21,1379$. https://doi.org/10.1364/JOSAB.21.001379

[47] Tsiatmas, A., Fedotov, V.A., García De Abajo, F.J. and Zheludev, N.I. (2012) Low-Loss Terahertz Superconducting Plasmonics. New Journal of Physics, 14, Article ID: 115006. https://doi.org/10.1088/1367-2630/14/11/115006

[48] Withayachumnankul, W. and Naftaly, M. (2014) Fundamentals of Measurement in Terahertz Time-Domain Spectroscopy. Journal of Infrared, Millimeter, and Terahertz Waves, 35, 610-637. https://doi.org/10.1007/s10762-013-0042-z

[49] Lee, D. and Cui, T. (2012) A Role of Silica Nanoparticles in Layer-by-Layer Self-Assembled Carbon Nanotube and $\mathrm{In}_{2} \mathrm{O}_{3}$ Nanoparticle Thin-Film pH Sensors: Tunable Sensitivity and Linearity. Sensors and Actuators A: Physical, 188, 203-211. https://doi.org/10.1016/j.sna.2012.01.004 